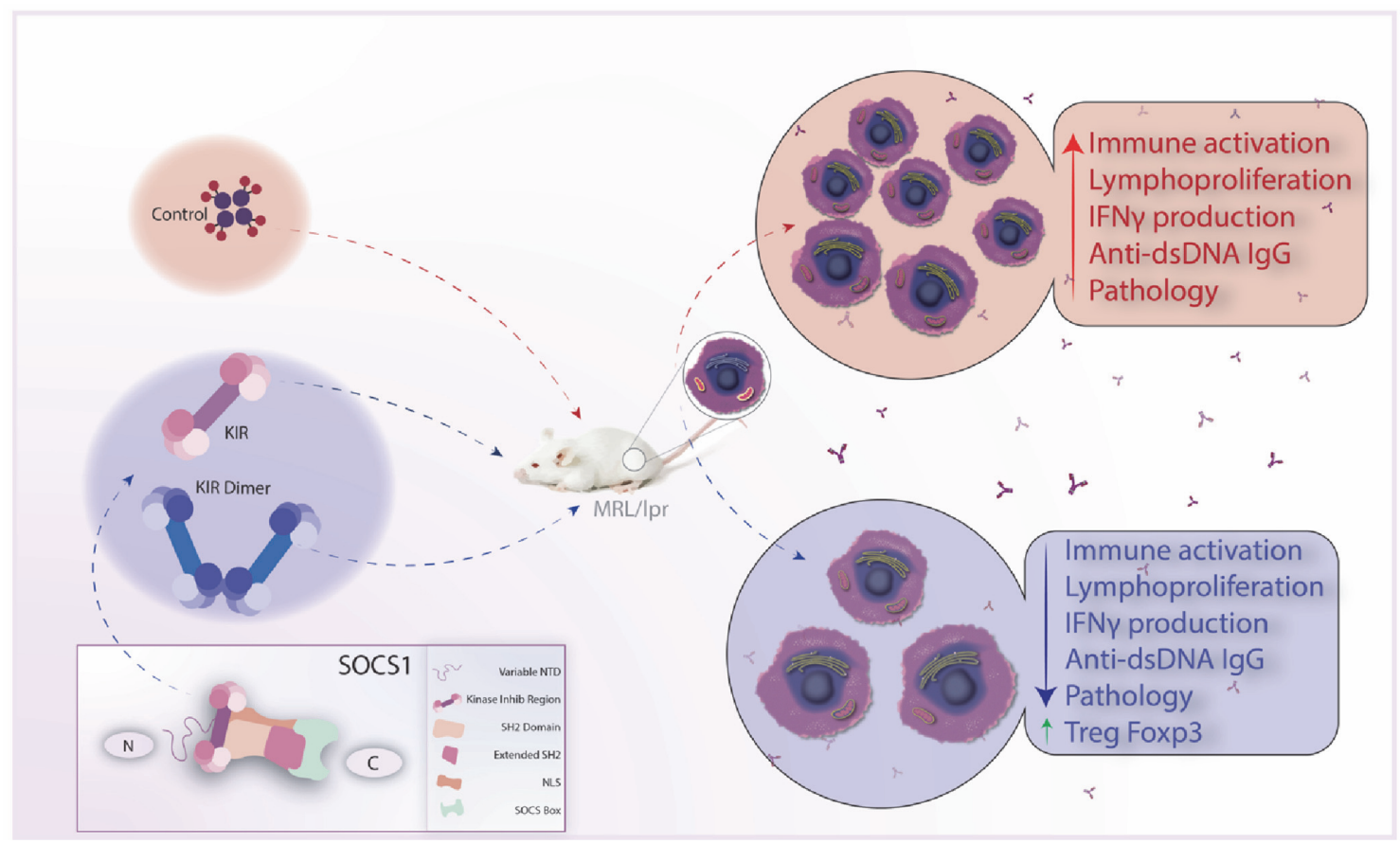

Abstract 1404 Figure 1 Graphical representation of the study design and results

Methods Female MRL/lpr mice received intraperitoneal injections of SOCS1-KIR peptide, or PBS carrier, 3 times per week and were monitored for lupus-like disease progression. Disease progression was based on the presence of skin lesions, lymphadenopathy, overall body score, and proteinuria. Peripheral blood, lymph nodes, and spleen was evaluated for peptide-mediated changes in lymphocyte populations by flow cytometry and qPCR. ELISA and western blot analysis were also employed to assess changes in lymphocyte activation. Finally, peptide mediated changes in renal pathology were analyzed.

Results We show that intraperitoneal administration of SOCS1KIR reduced the frequency, activation, and cytokine production of memory $\mathrm{CD}^{+}$and $\mathrm{CD}^{+} \mathrm{T}$ lymphocytes within the peripheral blood, spleen, and lymph nodes of treated mice. In addition, administration of SOCS1-KIR mimetic peptide treatment reduced lymphadenopathy, delayed the development and severity of skin lesions, reduced autoantibody production, and lupus associated kidney destruction. On a cellular level, SOCS1-KIR administration enhanced Foxp3 expression in both total splenic Tregs and follicular Tregs (figure 1). In addition, SOCS1-KIR treatment reduced the frequency of GL7+ germinal center enriched B cells and CD80+ leukocytes, which may potentially activate $\mathrm{T}$ lymphocytes.

Conclusion Together, these data show that SOCS1-KIR treatment was effective in reducing auto-reactive lymphocyte effector functions and suggest that therapeutic targeting of the SOCS1 pathway through peptide administration may have efficacy in mitigating lupus progression.

Acknowledgement We thank Mr. Benson and Dr. Moneypenny for flow cytometry assistance, UF animal care, and Drs. Hoffman and Wilson for technical expertise. This study was supported by the Lupus Research Institute, National Psoriasis Foundation, a BD Biosciences Research Grant, NIH/NCATS Clinical and Translational Science Awards TL1 TR000066 and UL1TR000064, NIH/NIAID subaward U01AI101990 and The Mcknight Fellowship Foundation.

\section{MECHANISMS UNDERLYING LUPUS SUPPRESSION MEDIATED BY MALE MICROBIOTA TRANSPLANTS IN FEMALE LUPUS-PRONE MICE}

James W Harder, Jing Ma, Pascale Alard, Michele M Kosiewicz*. University of Louisville, Louisville, KY, USA

\subsection{6/lupus-2021-lupus21century.82}

Background Sex and microbiota dysbiosis affect lupus development/progression. We have found that gut microbiota and metabolomic profiles in adult lupus-prone female and lupusresistant male NZBxNZWF1 (BWF1) mice differ; male cecal transplants into female mice suppress disease. Here, we identify disease-modulating bacteria and metabolite candidates and characterize potential mechanisms underlying disease suppression mediated by male microbiota transplants.

Methods BWF1 mice were used for all experiments. Fecal microbiota composition in female and male mice was determined by $16 \mathrm{~S}$ rRNA gene sequencing. Fecal metabolites were identified using mass spectrometry. Cecal contents from adult male or female mice were transplanted by oral gavage into female mice. Disease (proteinuria) and survival, and mesenteric

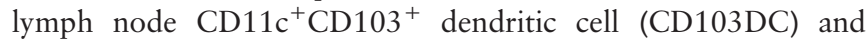
splenic $\mathrm{CD} 11 \mathrm{~b}^{+} \mathrm{F} 4 / 80^{+}$macrophage function in in vitro assays and by RNA-seq/RT-PCR were analyzed. In some experiments, female mice were treated with male fecal metabolites in vivo and cell function analyzed ex vivo, or female cells were treated directly with male metabolites in vitro and function analyzed.

Results Microbiota composition analysis in adult female and male mice and recipients of cecal transplants under conditions when male cecal transplants did or did not suppress disease indicated that high Bacteroides and low Clostridium abundances (high Bacteroides/Clostridium ratio) and presence of Alistipes correlated with disease resistance/suppression. Two types of immune cells with different functions were identified as possible mediators of sex-dependent and microbiota-driven 
differences in disease development. Female CD103DC have decreased ability to convert conventional CD4 cells to Foxp $3^{+}$ Tregs due to defects in retinoic acid (RA) production; female macrophages have decreased ability to mediate efferocytosis and defects in genes associated with PPAR $\square$ and LXR signaling. Male cecal transplants can restore function of both CD103DC and macrophages in female recipients. Metabolomics analysis of feces revealed that males have higher phytanic acid (PA), an RXR/PPAR $\square / L X R$ agonist. Treatment of female mice in vivo with $\mathrm{PA}$ delays disease to some extent and restores both CD103DC and macrophage function. PA also restores CD103DC and macrophage function when added to female cells directly in vitro. PA enhances CD103DC function by restoring RA production via the RXR pathway and macrophage efferocytosis via the PPAR $\square$ and LXR pathways in female mice.

Conclusions We have identified specific gut microbiota taxa and metabolite candidates from lupus-resistant male mice that have immunomodulatory activity and the potential to mitigate disease in lupus-prone female mice. Disease mitigation may be mediated through an increase in CD103DC function and macrophages efferocytosis.

Acknowledgments Funded by ALR and NIH-R01AR067188. 16S rRNA gene sequencing was performed by GTAC, Washington University, St. Louis. Mass spectrometry was performed by CREAM, University of Louisville, Louisville.

\section{ACELLULAR ARTIFICIAL ANTIGEN-PRESENTING CELLS INDUCE DISEASE-PROTECTIVE IMMUNOREGULATORY CELLS IN LUPUS}

${ }^{1}$ Antonio La Cava*, 'David A Horwitz. 'University of California Los Angeles, Los Angeles, CA, USA; ${ }^{2}$ General Nanotherapeutics, Santa Monica, CA, USA

\subsection{6/lupus-2021-lupus21century.83}

Background T regulatory cells (Tregs) provide beneficial effects in the suppression of autoimmune responses in systemic lupus erythematosus (SLE). We recently showed that poly(lactic-coglycolic acid) PLGA nanoparticles (NPs) loaded with IL-2 and TGF- $\beta$ could induce $\mathrm{CD}^{+}{ }^{+}$and $\mathrm{CD} 8^{+}$Foxp $3^{+}$Tregs in vitro and in vivo, with resulting protective effects in mice that develop lupus-like disease following the transfer of donor $\mathrm{T}$ cells from $\mathrm{DBA} / 2$ mice into $(\mathrm{C} 57 \mathrm{BL} / 6 \times \mathrm{DBA} / 2) \mathrm{F}_{1}$ (BDF1) recipients.

Methods We produced NPs encapsulating IL-2 as acellular tolerogenic artificial antigen-presenting cell (aAPC)s for the induction of $\mathrm{CD}^{+}$and $\mathrm{CD}^{+}{ }^{+} \mathrm{Foxp}^{+}$Tregs in vivo. aAPC NPs were also engineered to promote the tolerogenic activity of NK cells. Those acellular aAPCs were then tested for therapeutic efficacy in vivo in lupus-prone BDF1 mice.

Results The aAPC NPs induced $\mathrm{CD}^{+}$and $\mathrm{CD} 8^{+}$Tregs in vivo in BDF1 mice and protected the animals from lupus disease manifestations. At the NK cell level, protection from lupus-like disease could be ascribed to the production of TGF$\beta$ from the NP-induced NK cells. Finally, the use of aAPC NPs in humanized NSG mice expanded Tregs in numbers sufficient to protect mice from a rapidly evolving human antimouse graft-versus-host disease.

Conclusions These studies provide a proof-of-concept for the potential use of PLGA NPs as acellular aAPCs for the induction of therapeutically effective numbers of Tregs in conditions of pathologic deficit such as in SLE.

\section{0 - Genetics}

\section{GENETICS OF AGE AT SYSTEMIC LUPUS ERYTHEMATOSUS DIAGNOSIS}

${ }^{1}$ Raffaella Carlomagno, ${ }^{1}$ Fangming Liao, ${ }^{2}$ Jingjing Cao, 'Daniela Dominguez, ${ }^{3}$ Dafna D Gladman, ${ }^{4}$ Mariko Ishimori, ${ }^{4}$ Caroline Jefferies, ${ }^{5}$ Diane L Kamen, ${ }^{6}$ Sylvia Kamphuis, ${ }^{7}$ Marisa S Klein-Gitelman, ${ }^{1}$ Andrea M Knight, ${ }^{4}$ Chia-Chi I Lee, ${ }^{1}$ Deborah M Levy, ${ }^{8}$ Karen B Onel, ${ }^{2}$ Andrew D Paterson, ${ }^{9}$ Christine A Peschken, ${ }^{10}$ Janet E Pope, ${ }^{3}$ Zahi Touma, ${ }^{3}$ Murray B Urowitz, ${ }^{4}$ Daniel J Wallace, ${ }^{1}$ Declan Webber, ${ }^{3} J o a n$ E Wither, ${ }^{1,}{ }^{11}$ Earl D Silverman, ${ }^{1}$ ${ }^{2}$ Linda T Hiraki*. 'Division of Rheumatology; ${ }^{2}$ Genetics and Genome Biology, Research Institute, The Hospital for Sick Children; ${ }^{3}$ Schroeder Arthritis Institute, Krembil Research Institute, Toronto Western Hospital, Toronto, Canada; ${ }^{4}$ Division of Rheumatology, Department of Medicine, Cedars Sinai Medical Center, Los Angeles; ${ }^{5}$ Division of Rheumatology and Immunology, Medical University of South Carolina, Charleston, USA; ${ }^{6}$ Department of Pediatric Rheumatology, Sophia Children's Hospital, Erasmus University Medical Center, Rotterdam, Netherlands; 'Division of Rheumatology, Department of Pediatrics, Ann and Robert H. Lurie Children's Hospital of Chicago, Chicago; ${ }^{8}$ Pediatric Rheumatology, Hospital for Special Surgery, New York, USA; ${ }^{9}$ Departments of Medicine and Community Health Sciences, University of Manitoba, Winnipeg; ${ }^{10}$ Department of Medicine, University of Western Ontario, St. Joseph's Health Centre, London; ${ }^{11}$ Division of Translational Medicine Research Institute, The Hospital for Sick Children, Toronto, Canada

\subsection{6/lupus-2021-lupus21century.84}

Background Genome wide association studies (GWAS) have identified >100 SNPs associated with systemic lupus erythematosus (SLE) risk. There may be additional loci impacting the age of diagnosis. The purpose of this study was to identify genetic variants associated with age of SLE diagnosis.

Methods Our cohort included patients from tertiary care centres who met ACR and/or SLICC SLE classification criteria. We censored patients missing age at diagnosis. Patients were genotyped on the Illumina Multiethnic Array (MEGA) or Global Screen Array (GSA). Ungenotyped SNPs were imputed using the TopMed reference. We restricted to SNPs with a minor allele frequency (MAF) $\geq 0.01$ and imputation quality $\mathrm{R}^{2} \geq 0.3$. Ancestry was genetically inferred using principal components (PCs) and ADMIXTURE in reference to 1000 Genome Project. We completed genome-wide linear regression of log-transformed age at SLE diagnosis with GENESIS (significance $\left.\mathrm{P}<5 \times 10^{-8}\right)$. Multivariate models adjusted for sex and 5 PCs. We also conducted a GWAS of childhood-onset SLE

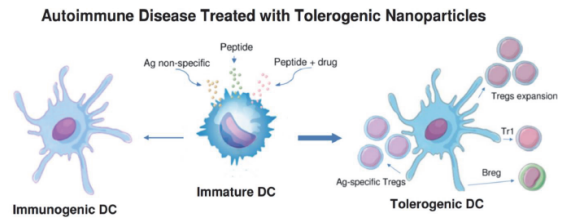

